\title{
PROPRIEDADES FÍSICAS DO SOLO APÓS EXTRAÇÃO SELETIVA DE MADEIRA NA AMAZÔNIA CENTRAL.
}

\author{
Sávio J. Filgueiras FERREIRA', Flávio J. LUIZÃO² ${ }^{2}$, Walane MELLO-IVOª , \\ Sheila M. ROSS ${ }^{4}$, Yvan BIOT ${ }^{5}$
}

RESUMO - Variáveis físicas do solo foram investigadas em parcelas de floresta de terra firme submetidas à extração seletiva de madeira na Amazônia central. Foram obtidas curvas de retenção de água no solo, juntamente com medidas de água disponivel no solo às plantas, densidade do solo e porosidade total. Medidas de temperatura do solo foram realizadas por mais de 13 meses, considerando seis tratamentos: controle, centro da clareira, borda da clareira, borda da floresta remanescente, floresta remanescente e trilha do trator. Medidas de condutividade hidráulica de solo saturado foram feitas na floresta e em clareiras, sem distinguir os tratamentos. O solo revelou baixa capacidade de armazenar água disponivel: apenas 11 a $18 \%$ da água pode estar disponivel às plantas, num perfil de $1 \mathrm{~m}$ de profundidade. A temperatura das camadas superiores do solo foi influenciada pela extração seletiva de madeira: nas clareiras abertas, a luz chega com mais intensidade no solo, proporcionando temperaturas mais elevadas no centro e nas bordas das clareiras do que no controle e na floresta remanescente.

Palavras-chave: Curva de retenção de água, temperatura do solo, condutividade hidráulica do solo, física do solo, extração seletiva de madeira.

\section{Soil Physical Properties after Selective Logging in Central Amazônia.}

ABSTRACT - Soil physical variables were investigated in forest plots submitted to selective logging in Central Amazonia. After logging, soil samples were collected to obtain soil water retention curves and measure: available soil water to the plants, soil density, and total porosity. Temperature measurements were carried out for 13 months, considering six treatments: control, center of the gaps, edge of the gaps, edge of the remaining forest, remaining forest and tractor tracks. Hydraulic conductivity measurements on saturated soil were conducted both on the control forest as well as on the logged plots, with no treatment distinction. The soil showed a low available water storage capacity: only 11 to $18 \%$ can be available to the plants, up to 1 meter depth. The temperature of the soil upper layers was influenced by logging, i. e., through the opening of the gaps, light reaches the soil more intensely in the center and edge of gaps, increasing temperatures in relation to the control and the remaining forest.

Key-words: Water retention curve, soil temperature, hydraulic conductivity, soil physics, selective logging.

\section{Introdução}

De modo geral, o solo sob floresta de terra firme da Amazônia é caracterizado por sua baixa fertilidade química, sendo a sustentação da floresta garantida pela ciclagem, praticamente fechada, de nutrientes, aliada às condições de calor, alta umidade e alta diversidade biológica. Por isso na terra firme da Amazônia (aproximadamente $90 \%$ da área) o potencial agropecuário é baixo. Por outro lado, o potencial madeireiro é

'Coordenação de Pesquisas em Geociências/CPGC, INPA, C. Postal 478, CEP 69011-970, Manaus, AM. ${ }^{2}$ Coordenação de Pesquisas em Ecologia/CPEC, INPA, C. Postal 478,CEP: 69011-970, Manaus, AM. ${ }^{3}$ EMBRAPA Tabuleiros Costeiros/ERP de Rio Largo. C. Postal 2013. CEP: 57061-970, Aracaju, SE. ${ }^{4}$ U. K. Environmental Research \& Consultancy (ERC), University of Liverpool - England.

${ }^{5}$ Department for International Development (DFID, Jl Jend. Gatot Subroto, Jakarta 10270 Indonesia. 
elevado, mas a falta de informações sobre o manejo dessas áreas limita as perspectivas de sustentabilidade do ecossistema.

Até a década de 60 , as estruturas econômica e social da Amazônia brasileira foram fortemente influenciadas pelo extrativismo florestal, principalmente de borracha. A partir dessa década começou o desmata-mento em grande escala, para plantios agricolas ou pastagens, que tem se concentrado em um arco que vai do Estado do Pará, no leste, passando pelo Mato Grosso, até Rondônia, no oeste (Uhl \& Almeida, 1996). A diminuição do estoque madeireiro das regiões Sul e Sudeste também tem contribuido para o desmatamento, geralmente descon-trolado e predatório, trazendo sérias preocupações sobre a capacidade de recuperação do solo e da floresta em si (Nortcliff \& Dias, 1988; Uhl \& Almeida, 1996).

Na região de Manaus (Amazônia central), em área de Latossolo Amarelo de textura muito argilosa, estudos sobre o manejo florestal sustentável têm sido desenvolvidos, adotando o sistema S.E.L. (Seleção de Espécies Listadas). Este sistema consiste na extração seletiva de madeira de espécies já com valor comercial ou com valor potencial devido às suas características físicas ou silviculturalmente importantes (Higuchi et al., 1991). Um manejo florestal sustentado poderia ser obtido apenas pela condução do povoamento florestal natural ao longo de um determinado tempo, isto é, pelo aproveitamento daquilo que a própria floresta produz, pela regeneração natural, sem comprometer o seu capital inicial (Higuchi et al., 1991).

No período de 1993-97, o Projeto "Biomassa e Nutrientes na Floresta Tropical Úmida - Projeto "BIONTE", financiado pelo DFID (Department for International Development) do Reino Unido, estudou interdisciplinarmente a influência de uma extração seletiva de madeira, de nivel moderado, sobre o solo e a vegetação, com o intuito de avaliar e definir as estratégias para futuros empreendimentos madeireiros em áreas de floresta de terra firme. Este trabalho é parte deste projeto, e teve como objetivo avaliar as alterações fisicas do solo, causadas pela extração seletiva de madeira.

\section{Materiais e Métodos}

Local do estudo. $O$ estudo foi realizado na Estação de Manejo Florestal do INPA, cerca de $80 \mathrm{~km}$ ao norte de Manaus, em uma área localizada entre os quilômetros 21 e 24 da margem esquerda da estrada vicinal ZF-2 (Fig. 1). As coordenadas geográficas dessa área são as seguintes: $02^{\circ} 37^{\prime}$ a $02^{\circ} 38^{\prime}$ de latitude Sul e $60^{\circ} 09^{\prime}$ a $60^{\circ} 11^{\prime}$ de longitude Oeste.

Geologicamente, o solo da área deste estudo faz parte da Sinéclise do Amazonas, em cujas margens uma sequência sedimentar foi depositada, no Terciário, formando o que se denomina de Grupo das Barreiras (Vieira \& Santos, 1987). O solo da área de estudo é um "Latossolo Amarelo, álico, textura muito argilosa" (Camargo \& Rodrigues, 
1979; Chauvel et al., 1992). É composto de caulinita $(80 \%)$, hidróxido de ferro e alumínio, e de 5 a $15 \%$ de quartzo. Chauvel (1982) estudou o solo da área e estratificou o perfil em seis horizontes: $\mathrm{O}-2-0 \mathrm{~cm}$, A1 - 0-5 cm, A3 - 5-35 cm, B1 - 35$70 \mathrm{~cm}, \mathrm{~B} 21-70-150 \mathrm{~cm}$ e B22 - 150 $230 \mathrm{~cm}$. O teor de argila situou-se entre 80,5 e $89,1 \%$.

\section{Delineamento experimental.} Para este estudo, foram investigadas três parcelas controle (floresta intacta) e três que sofreram extração seletiva de madeira em três blocos casualizados; cada parcela tinha $200 \mathrm{~m}$ x $200 \mathrm{~m}$. O corte ocorreu em meados de setembro de 1993 , com a retirada de 6-10 árvores/ha (em média, $34 \mathrm{~m}^{3}$ $\mathrm{ha}^{-1}$ ), pertencentes a espécies com potencial madeireiro, as chamadas "Espécies Listadas", com um DAP (diâmetro à altura do peito) maior de $50 \mathrm{~cm}$. As árvores foram cortadas com motosserra e retiradas do local por um trator Caterpillar D6. Logo após o corte (novembro e dezembro), em cada parcela, foi aberta uma trincheira para a coleta de amostras de solo; nas parcelas manejadas, as trincheiras foram abertas no centro de uma clareira e nas parcelas-controle foram abertas próximo ao hectare central. Foram investigadas as seguintes variáveis: curvas de retenção da água no solo, água disponivel no solo ou a capacidade do reservatório às plantas, densidade do solo, porosidade total.

As medidas de condutividade hidráulica do solo foram realizadas nos três blocos, nas parcelas-controles e nas manejadas. No bloco IV foi acrescentada uma parcela que sofreu extração seletiva de madeira, com intensidade similar de corte, em 1987. Dentro desta parcela fizeram-se medidas em dois locais, sendo uma onde foi constatada a retirada de árvores e outra na floresta remanescente, que considerou-se como um controle; portanto, no total, foram investigadas quatro parcelas-controle e quatro parcelas manejadas. Para as determinações foram escolhidos pontos ao acaso no hectare central.

O corte seletivo produziu cinco classes de distúrbios: trilha do trator (TT), centro da clareira (CC), borda da clareira com a floresta remanescente $(\mathrm{BC})$, borda da floresta remanescente com a clareira (BF) e floresta remanescente (FR). Parcelas-controle, que não sofreram extração seletiva, foram incluídas como o sexto tratamento. Nestes tratamentos do corte de 1993 e após cinco meses do corte, foi estudada a temperatura do solo. Os tratamentos foram repetidos em três blocos (I, II e IV), sendo que um quarto bloco (III) não foi manejado por apresentar solos e topografia diferentes dos três outros blocos.

Curvas de retenção de água do solo, densidade e porosidade. Amostras de solo foram coletadas usando-se cilindros volumétricos com um dos bordos cortante, não deformadas, em cada parcela, controle e manejada (centro da clareira), nas profundidades $0-5,10,20,40,60 \mathrm{e}$ $100 \mathrm{~cm}$ de profundidade. Para a primeira profundidade empregou-se 0 $5 \mathrm{~cm}$ por coincidir com a altura dos 


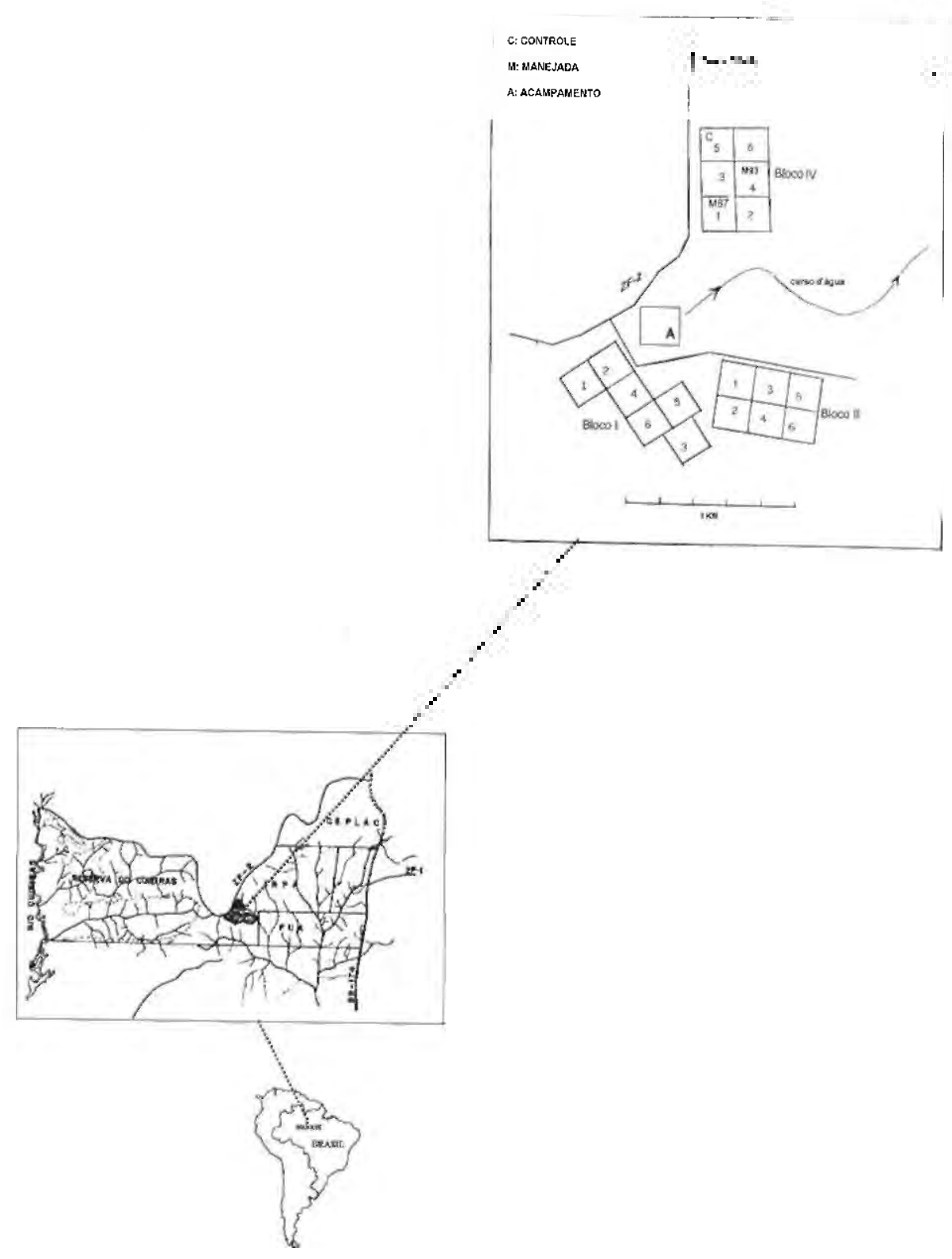

Figura 1. Área de estudo do manejo florestal (hachuriada) com a distribuição dos blocos (I, II e IV), divididos em parcelas (1 a 6). C=controle; M87 parcela submetida à extração seletiva em 1987; M93= extraçào seletiva em 1993. 
cilindros, enquanto nas demais o centro da altura do cilindro volumétrico correspondeu à profundidade considerada. As curvas de retenção de água do solo foram determinadas de forma clássica, utilizando-se a mesa de tensão para as tensões de 0 (saturada), 0,1, 0,4 e 0,6 $\mathrm{kPa}$ e a câmara de Richard para as tensões de $10,30,100,200,500 \mathrm{e}$ $1500 \mathrm{kPa}$. Após cada equilíbrio obtido, as amostras eram retiradas do aparelho e pesadas, sendo então as amostras secas em estufa a $105-110^{\circ} \mathrm{C}$, e novamente pesadas para determinar as massas de sólidos. A porosidade do solo ou volume total de poros total, foi tomada como sendo a umidade q para a tensão 0 (saturada). Para o ajuste das curvas de retenção foi empregado o Programa SWRC ("CURVARET", versão $2.00 \mathrm{p}$ ) de autoria de DouradoNeto et al. (1996), onde os potenciais mátricos $\left(\left|\Psi_{\mathrm{m}}\right|\right.$, em $\mathrm{cm}$ de coluna de água) foram relacionados com as umidades $\theta$ do Modelo Matemático Empírico de Van Genuchten (1980), dado pela equação:

$$
\theta=\theta_{r}+\frac{\left(\theta_{s}-\theta_{r}\right)}{\left[1+\left(\alpha\left|\psi_{m}\right|\right)^{n}\right]^{m}}
$$

onde: q é a umidade à base de volume $\left(\mathrm{cm}^{3} / \mathrm{cm}^{3}\right),\left|\psi_{\mathrm{m}}\right|$ o módulo do potencial mátrico ( $\mathrm{cm}$ de água), $\theta_{\mathrm{r}}$ é a umidade residual $\left(\mathrm{cm}^{3} / \mathrm{cm}^{3}\right), \theta_{\mathrm{s}}$ a umidade de saturação $\left(\mathrm{cm}^{3} / \mathrm{cm}^{3}\right) ; \alpha, \mathrm{m} \mathrm{e} \mathrm{n}\left(\mathrm{cm}^{-1} \mathrm{de}\right.$ água) são parâmetros empíricos obtidos do modelo pela regressão (Tab. 1).

Com os dados ajustados de umidade, foi possivel calcular a água disponivel (AD), determinada entre as tensões 10 e $1500 \mathrm{kPa}$, ou seja, entre a capacidade de campo (CC) e o ponto de murcha permanente (PMP). Geralmente é empregada a tensão 30 $\mathrm{kPa}(1 / 3 \mathrm{~atm})$, mas, segundo Reichardt (1988), deve-se empregar tensão mais baixa, isto é, potencial mais alto, da ordem de -10 a $-6 \mathrm{kPa}$, o que foi feito neste trabalho. Para o cálculo da água disponivel foi utilizada a seguinte equação:

$$
\mathrm{AD}(\mathrm{cm})=\left(\theta_{10}-\theta_{1500}\right)
$$

onde $\theta_{100}$ e $\theta_{15000}$ são respectivamente as umidades volumétricas obtidas entre a capacidade de campo e o ponto de murcha permanente. Levando-se em conta a profundidade, a equação fica:

$$
\mathrm{AD}(\mathrm{cm})=\left(\theta_{10}-\theta_{1500}\right) \mathrm{h}
$$

sendo $\mathbf{h}$ a profundidade.

Condutividade hidráulica de solo saturado. A condutividade hidráulica saturada ou infiltração básica Ko $\left(\mathrm{mm} . \mathrm{h}^{-1}\right)$ foi determinada no campo, utilizando-se o permeâmetro de Guelph, modelo 2800, da Soil Moisture Equipment Corp.. Este aparelho funciona com carga constante, que é mantida por um sistema cujo princípio é o frasco de Mariotte. O sistema instrumental é constituído principalmente de um tripé como suporte, um tubo suporte, um reservatório cilíndrico e um conjunto de tubos que servem para estabelecer o fluxo (registro) e medir a carga hidráulica. Este método foi proposto por Reynolds \& Elrick (1985), estando também descrito em Reynolds (1993).

Temperatura do solo. Nas 
parcelas-controle, foram instalados dois conjuntos de termômetros de mercúrio, com suporte metálico, a 5 e a $10 \mathrm{~cm}$ de profundidade do solo, em locais (posições) escolhidos ao acaso dentro do hectare central da parcela. Nas parcelas manejadas, um conjunto de termômetros foi instalado no centro da clareira, onde foi aberta a trincheira. Com exceção da borda da clareira do bloco II, onde foram instalados termômetros nas duas profundidades, em um transecto, a partir do centro da clareira, foram instalados termômetros (somente a 5 $\mathrm{cm}$ de profundidade) nos tratamentos: trilha do trator, centro da clareira, borda da clareira, borda da floresta remanescente e floresta remanescente, totalizando, com a floresta controle, seis tratamentos em cada bloco. As leituras foram feitas geralmente entre $11: 00$ e $14: 00 \mathrm{hs}$, no periodo de $10 / 02 /$ 94 até $21 / 02 / 95$, geralmente com intervalo semanal entre as medidas. Usando-se os dados de temperatura nas duas profundidades $(5$ e $10 \mathrm{~cm})$, calculou-se o gradiente de temperatura que, juntamente com a condutividade térmica do solo, é importante para a determinação da taxa de fluxo de calor no solo. $\mathrm{O}$ gradiente de temperatura foi calculado pela equação:

$$
\text { gradiente } T=\frac{\partial T}{\partial \mathbf{z}}
$$

onde $\mathbf{T}$ é a temperatura $\left({ }^{\circ} \mathrm{C}\right)$ e $\mathbf{z}$ a coordenada de posição $(\mathrm{cm})$. Quando o gradiente de temperatura é negativo indica que a temperatura do solo é mais elevada na camada superficial do que na camada mais profunda.

\section{Resultados e Discussão}

Curvas de retenção, porosidade total e densidade. As curvas de retenção da água no solo, além de permitirem a obtenção indireta da umidade do solo por meio de tensiômetro, permitiram observar uma capacidade maior de água volumétrica e também de disponibilidade de água às plantas na camada superior do solo (até $10 \mathrm{~cm}$ de prof.), tanto nas parcelascontrole como nas manejadas pela extração seletiva de madeira (Fig. 2).

As curvas referentes às camadas mais superficiais apresentam um comportamento diferente das outras camadas, podendo-se observar que elas cruzam as demais. Isto indica que, com umidade elevada, essas camadas tendem a fornecer mais água quando submetidas a tensões em torno de 100 $\mathrm{kPa}$. As camadas mais profundas não apresentam essa característica em suas curvas e, portanto, dispõem de menos água para as plantas. Possivelmente o teor de matéria orgânica, a densidade mais baixa e a menor quantidade de argila nas camadas superiores sejam responsáveis por este comportamento do solo. As curvas de retenção obtidas por Tomasella \& Hodnett (1996), também na região de Manaus, em condições de campo, mostraram comportamento semelhante, principalmente em relação às camadas mais profundas. O mesmo comportamento foi ainda observado nas curvas de retenção obtidas por Corrêa (1984) em Latossolo Amarelo, na Amazônia central, que a partir da tensão de $200 \mathrm{kPa}$, verificou que essas 

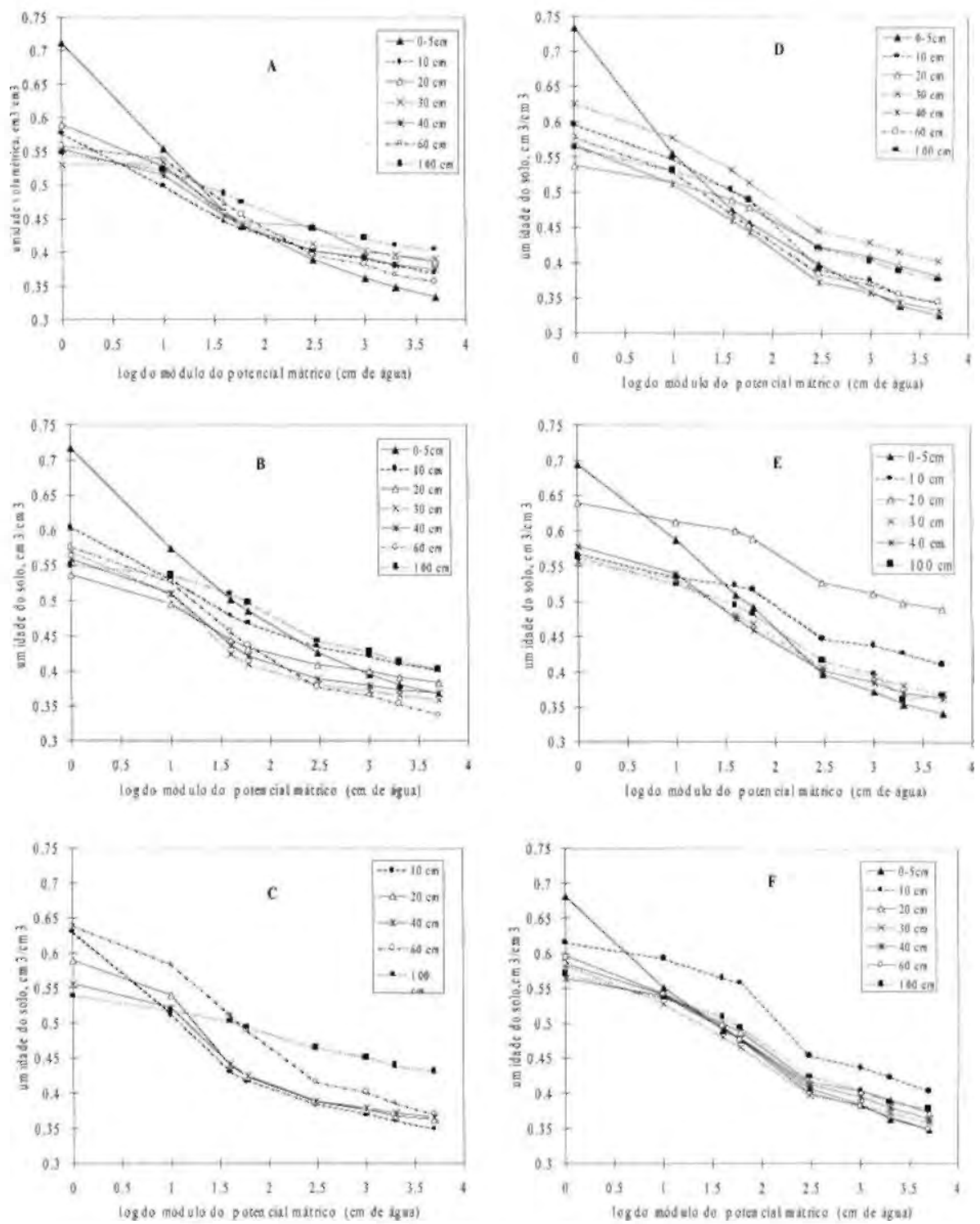

Figura 2. Curvas de retenção da água no solo das parcelas controle (à esquerda) dos Blocos I (A), II (B) e IV (C) e das parcelas manejadas (à direita) dos Blocos I (D), II (E) e IV (F) cm seis profundidades: $0-5,10,20,40,60$ e $100 \mathrm{~cm}$. 
curvas tendem à horizontalidade.

Os resultados de água disponível $(\mathrm{AD}, \mathrm{cm})$ não levam em conta a camada de solo, mas somente a variação da umidade entre os potenciais -10 e $-1500 \mathrm{kPa}$ (Tab. 1); também são fornecidos os valores correspondentes aos parâmetros empíricos $(\alpha, \mathbf{m}$ e $\mathbf{n})$ obtidos por regressão do modelo de Van Genuchten (1980). Os valores mais elevados de AD foram encontrados nas camadas superficiais e tendem a diminuir até os $40 \mathrm{~cm}$ do solo; depois, os valores tornam a aumentar com a profundidade do solo. Os resultados variaram de 0,04 a $0,13 \mathrm{~cm}$. Estudos anteriores das características físicas de alguns solos de terra firme próximos a Manaus, mostraram que os Latossolos Amarelos argilosos geralmente têm baixa capacidade de reter água disponível (Reichardt et al., 1980). O valor médio obtido por eles para um mesmo tipo de solo foi de $2,00 \mathrm{~cm}$ para uma camada de $30 \mathrm{~cm}$, correspondendo a $0,67 \mathrm{~cm}$ para a camada de $10 \mathrm{~cm}$. Os autores consideraram como água disponivel a água retida pelo solo entre as tensões de 30 e $1500 \mathrm{kPa}$. Dados de AD entre as tensões 10 e $1500 \mathrm{kPa}$, fornecidos por Corrêa (1984), foram da ordem de $0,11 \mathrm{~cm}$ para a camada superficial $(0$ $8 \mathrm{~cm}$ ) de um Latossolo Amarelo próximo a Manaus. Em solo de várzea, na ilha do Careiro (Amazônia central), foram registrados valores bem mais elevados de $A D$, entre 0,45 a $0,15 \mathrm{~cm}$, para a camada de solo 0 $10 \mathrm{~cm}$, entre as tensões 10 e $1500 \mathrm{kPa}$ (Ferreira, 1993).
O valor mais elevado de umidade saturada $\left(\theta_{\mathrm{s}}\right)$, que corresponde ao espaço poroso, ou porosidade, foi obtido na profundidade de solo de $5 \mathrm{~cm}$, na parcela manejada do bloco I $\left(0,73 \mathrm{~cm}^{3} / \mathrm{cm}^{3}\right)$, indicando uma porosidade muito elevada $(73,4 \%)$. Os valores mais elevados de umidade saturada foram obtidos nas camadas superficiais do solo, tendendo a diminuir com a profundidade (Tab. 1); mesmo assim, as camadas mais profundas apresentaram porosidade acima de 50 $\%$. Verifica-se ainda que o conteúdo de água retido no potencial $-1500 \mathrm{kPa}$ é relativamente elevado, variando de 0,31 a $0,40 \mathrm{~cm}^{3} / \mathrm{cm}^{3}$. Corrêa (1984) registrou valor mínimo de $0,26 \mathrm{~cm}^{3} /$ $\mathrm{cm}^{3}$ em Latossolo Amarelo para o mesmo potencial mátrico. No entanto, Ferreira (1993) obteve valores bem mais baixos, de 0,05 a $0,31 \mathrm{~cm}^{3} / \mathrm{cm}^{3}$, em amostras de solo de várzea do Amazonas.

As estimativas acumuladas de porosidade total (capacidade máxima de armazenamento de água) para a camada de solo até $1 \mathrm{~m}$ de profundidade, em média, estão em torno de $57 \mathrm{~cm}$; para a água disponivel às plantas (AD) seriam de 6,4 a 10,6 $\mathrm{cm}$, e para a água retida em potencial abaixo de $-1500 \mathrm{kPa}$, mais de $36 \mathrm{~cm}$ (Tab. 2). Abaixo deste potencial, as plantas não conseguem retirar água do solo e esta quantidade chega a ser mais de três vezes maiores do que da AD. Tais estimativas revelaram que, quando saturado, dos cerca de $60 \%$ de água que o solo saturado pode reter, quase $2 / 3$ não podem ser retirados 
Tabela 1. Valores dos parâmetros de ajuste das relações entre o potencial mátrico c a umidade do solo pelo modelo de Van Genuchten (1980) nas parcelas-controle e manejadas em 1993.

\begin{tabular}{|c|c|c|c|c|c|c|c|c|c|}
\hline Local & $\begin{array}{l}\text { Prof } \\
(\mathrm{cm})\end{array}$ & $\alpha$ & $\mathrm{m}$ & $\mathrm{n}$ & $\theta_{s}$ & $a_{r}$ & $0_{10}$ & $0_{1500}$ & $\begin{array}{c}A D \\
(\mathrm{~cm})\end{array}$ \\
\hline \multicolumn{10}{|c|}{ Parcelas-controle } \\
\hline Bloco I & 0.5 & 0,3968 & 0,2487 & 1,3310 & 0,71 & 0,30 & 0,42 & 0,32 & 0,10 \\
\hline Bloco II & 0.5 & 0,5142 & 0.1979 & 1,2467 & 0,72 & 0,31 & 0.46 & 0,35 & 0,11 \\
\hline Bloco I & 10 & 0,3799 & 0,2226 & 1,2863 & 0.58 & 0,34 & 0,42 & 0,36 & 0,06 \\
\hline Bloco II & 10 & 0.3368 & 0,2318 & 1,3018 & 0,60 & 0,38 & 0,46 & 0,40 & 0,06 \\
\hline Bloco IV & 10 & 0,3123 & 0,2967 & 1,4218 & 0,63 & 0,33 & 0,40 & 0,34 & 0,06 \\
\hline Bloco I & 20 & 0.2760 & 0,2371 & 1,3108 & 0,59 & 0,36 & 0,44 & 0,38 & 0,06 \\
\hline Bloco II & 20 & 0,1610 & 0,2934 & 1,4152 & 0,54 & 0,37 & 0,42 & 0,38 & 0,04 \\
\hline Bloco IV & 20 & 0,0931 & 0,4179 & 1,7180 & 0,59 & 0,36 & 0.40 & 0,36 & 0,04 \\
\hline Bloco I & 30 & 0,0443 & 0,4687 & 1,8822 & 0,53 & 0,39 & 0,43 & 0,39 & 0,04 \\
\hline Bloco II & 30 & 0.1203 & 0.4009 & 1,6692 & 0,57 & 0.36 & 0,40 & 0,36 & 0,04 \\
\hline Bloco 1 & 40 & 0,1071 & 0,3207 & 1,4721 & 0,55 & 0,36 & 0,43 & 0,37 & 0.06 \\
\hline Bloco If & 40 & 0.1083 & 0,3896 & 1,6382 & 0,56 & 0,36 & 0,41 & 0,37 & 0,04 \\
\hline Bloco IV & 40 & 0,0817 & 0,4026 & 1,6739 & 0,55 & 0,36 & 0,41 & 0,36 & 0,05 \\
\hline Bloco I & 60 & 0,0580 & 0,3159 & 1,4618 & 0.56 & 0,34 & 0,43 & 0,35 & 0,08 \\
\hline Bloco II & 60 & 0,1124 & 0,2799 & 1,3887 & 0,58 & 0,32 & 0,42 & 0,33 & 0,09 \\
\hline Bloco IV & 60 & 0.1072 & 0,2695 & 1,3689 & 0,64 & 0,34 & 0,46 & 0,36 & 0.10 \\
\hline Bloco I & 100 & 0,0851 & 0,2663 & 1,3447 & 0,55 & 0,38 & 0,46 & 0,40 & 0,06 \\
\hline Bloco II & 100 & 0,0379 & 0,2638 & 1,3583 & 0,55 & 0,37 & 0,48 & 0,39 & 0.09 \\
\hline Bloco IV & 100 & 0,1254 & 0,1180 & 1,1338 & 0,54 & 0,35 & 0,48 & 0,42 & 0,06 \\
\hline \multicolumn{10}{|c|}{ Parcelas com extração seletiva de } \\
\hline Bloco I & $0-5$ & 0,7321 & 0,1818 & 1,2222 & 0,73 & 0,25 & 0.44 & 0.31 & 0,13 \\
\hline Bloco Il & 0.5 & 0,2000 & 0,2281 & 1,2955 & 0,69 & 0,29 & 0,46 & 0,33 & 0,13 \\
\hline Bloco IV & 0.5 & 0,5822 & 0,1618 & 1,1931 & 0,68 & 0,26 & 0.45 & 0,33 & 0,12 \\
\hline Bloco I & 10 & 0,0718 & 0,2503 & 1,3338 & 0,60 & 0,30 & 0,45 & 0,33 & 0,12 \\
\hline Bloco II & 10 & 0,0684 & 0,1814 & 1,2216 & 0,57 & 0,35 & 0,49 & 0,40 & 0.09 \\
\hline Bloco IV & 10 & 0,0261 & 0,3191 & 1,4687 & 0,61 & 0,38 & 0,52 & 0,39 & 0,13 \\
\hline Bloco 1 & 20 & 0,0629 & 0.2015 & 1,2524 & 0,54 & 0,33 & 0,46 & 0,37 & 0.09 \\
\hline Bloco II & 20 & 0,0417 & 0,2600 & 1,3513 & 0,64 & 0,46 & 0.57 & 0,48 & 0,09 \\
\hline Bloco IV & 20 & 0,1536 & 0,1884 & 1,2322 & 0,60 & 0,31 & 0,46 & 0,36 & 0,10 \\
\hline Bloco I & 30 & 0,1123 & 0,2266 & 1,2929 & 0,63 & 0,36 & 0,49 & 0,39 & 0,10 \\
\hline Bloco II & 30 & 0,0660 & 0,2703 & 1,3705 & 0,56 & 0,34 & 0,45 & 0,36 & 0,09 \\
\hline Bloco IV & 30 & 0,1371 & 0,2287 & 1,2966 & 0,58 & 0,32 & 0,44 & 0,35 & 0,09 \\
\hline Bloco I & 40 & 0.1239 & 0,2399 & 1,3157 & 0,57 & 0,30 & 0.42 & 0,32 & 0,10 \\
\hline Bloco II & 40 & 0,0892 & 0,2877 & 1,4039 & 0,58 & 0,34 & 0,44 & 0,36 & 0,08 \\
\hline Bloco IV & 40 & 0,0633 & 0,2411 & 1,3176 & 0,56 & 0,33 & 0,46 & 0,35 & 0,11 \\
\hline Bloco I & 60 & 0,1051 & 0,2666 & 1,3635 & 0.58 & 0,32 & 0,43 & 0.33 & 0,10 \\
\hline Bloco IV & 60 & 0,0927 & 0,2196 & 1,2818 & 0,58 & 0,30 & 0,45 & 0,34 & 0.11 \\
\hline Bloco I & 100 & 0,0610 & 0,2260 & 1,2919 & 0,56 & 0,33 & 0,47 & 0,36 & 0,11 \\
\hline Bloco II & 100 & 0,0724 & 0.2248 & 1,2899 & 0.56 & 0,32 & 0,46 & 0,36 & 0,10 \\
\hline Bloco IV & 100 & 0,0617 & 0,2346 & 1,3066 & 0,57 & 0,34 & 0.47 & 0,37 & 0,10 \\
\hline
\end{tabular}


pelas plantas, pois está retida em potenciais a partir de $-1500 \mathrm{kPa}$; em torno de $20 \%$ pode ser perdida pela ação da gravidade e somente cerca de 11 a $18,5 \%$ de água desses solos podem estar disponíveis às plantas. Considerando que o solo atinja a saturação e subtraindo-se da porosidade total a $\mathrm{AD}$ e a água retida em potencial abaixo de $-1500 \mathrm{kPa}$, nota-se que a diferença varia de 10,4 a $14,6 \mathrm{~cm}$, que corresponde à água a ser perdida pela ação da gravidade. Para que ocorra a saturação do solo em condições de campo, é necessária que a taxa de entrada de água seja superior à de infiltração, que mostrou ser muito alta. Durante um período de aproximadamente dois anos, não foi possivel observar acúmulo de água na superfície, ou escoamento superficial, que pudesse indicar saturação de água do solo.

Nas parcelas manejadas, os valores de disponibilidade de água foram superiores em relação às parcelascontrole. Ou seja, uma modificação na distribuição de tamanho de poros deve ter ocorrido, mesmo que a porosidade tenha permanecido a mesma, indicando que a extração seletiva de madeira não causou diminuição no volume total de poros, tampouco na retenção de água abaixo do potencial mátrico $-1500 \mathrm{kPa}$, isto é, na disponibilidade de água às plantas. Assim, essas caracteristicas não foram significativamente alteradas devido à extração seletiva de madeira nas parcelas estudadas. No entanto, é importante salientar que as amostras nas parcelas manejadas foram retiradas no centro de clareiras e não em áreas atingidas por distúrbios mais severos, como nas trilhas de trator. Além disso, eventuais diferenças podem ter ocorrido na distribuição de macro-poros, mesmo no caso em que a porosidade total não mostrou diferenças (Grimaldi et al., 1993).

Os valores menores de densidade foram obtidos nas camadas superficiais, tanto das parcelascontrole quanto nas que sofreram extração de madeira, onde foi encontrado o valor mais baixo, de 0,67 $\mathrm{g} / \mathrm{cm}^{3}$ no bloco I (Tab. 3). Foi observada a tendência de aumento da densidade com a profundidade do solo, tanto no controle como no tratamento com extração seletiva. $\mathrm{O}$ valor mais elevado foi $1,23 \mathrm{~g} / \mathrm{cm}^{3}$, obtido na parcela controle do bloco II a $1 \mathrm{~m}$ de profundidade. Entre 10 e 30 $\mathrm{cm}$ de profundidade, foram encontrados valores mais elevados de densidade do solo nas parcelas manejadas do que no controle, especialmente a $10 \mathrm{~cm}$ de profundidade nos blocos II e IV, o que pode indicar uma leve compactação sub-superficial.

$$
\text { Nortcliff \& Dias }
$$
obtiveram valores semelhantes aos do presente trabalho, para floresta virgem próximo a Manaus $\left(0,79 \mathrm{~g} / \mathrm{cm}^{3}\right.$ para a camada $0-5 \mathrm{~cm})$, indicando que a densidade aumentava com a profundidade. Observaram que os dados obtidos em floresta intacta em relação à que sofreu desmatamento apresentou pouca alteração. Os resultados do presente estudo foram mais baixos do que aos obtidos por 
Tabela 2. Estimativa de acumulação da porosidade total, água disponivel (AD) e água retida $\mathrm{em}$ potencial abaixo de $-1500 \mathrm{kPa}$ ate $1 \mathrm{~m}$ de profundidade, nas parcelas-controle e nas extraidas seletivamente.

\begin{tabular}{|c|c|c|c|c|c|}
\hline & Prof $(\mathrm{cm})$ & $\begin{array}{l}\text { Espessura da } \\
\text { Camada }(\mathrm{cm})\end{array}$ & $\begin{array}{l}\text { Porosidade } \\
\text { Acumulada }\end{array}$ & $\begin{array}{c}A D \\
\text { acumulada }\end{array}$ & $\begin{array}{l}-1500 \mathrm{kPa} \\
\text { Acumulada }\end{array}$ \\
\hline \multicolumn{6}{|c|}{ Parcelas-controle } \\
\hline \multirow[t]{7}{*}{ Bloco I } & 0.5 & 5 & 3,6 & 0,5 & 1,6 \\
\hline & 10 & 5 & 6.4 & 0,8 & 3,4 \\
\hline & 20 & 10 & 12.3 & 1,4 & 7,2 \\
\hline & 30 & 10 & 17,6 & 1,8 & 11,1 \\
\hline & 40 & 10 & 23,2 & 2,4 & 14,8 \\
\hline & 60 & 20 & 34,3 & 4.0 & 21,8 \\
\hline & 100 & 40 & 56.2 & 6,4 & 37,8 \\
\hline \multirow[t]{7}{*}{ Bloco ॥ } & $0-5$ & 5 & 3,6 & 0,6 & 1,8 \\
\hline & 10 & 5 & 6,6 & 0.9 & $3 ; 8$ \\
\hline & 20 & 10 & 12,0 & 1,3 & 7,6 \\
\hline & 30 & 10 & 17,7 & 1,7 & 11,2 \\
\hline & 40 & 10 & 23.3 & 2,1 & 14,9 \\
\hline & 60 & 20 & 34.8 & 3,9 & 21,5 \\
\hline & 100 & 40 & 56.8 & 7,5 & 37,1 \\
\hline \multirow[t]{5}{*}{ Bloco IV } & 10 & 10 & 6,3 & 0,6 & 3.4 \\
\hline & 20 & 10 & 12.2 & 1,0 & 7,0 \\
\hline & 40 & 20 & 23,3 & 2.0 & 14,2 \\
\hline & 60 & 20 & 36,1 & 4,0 & 21,4 \\
\hline & 100 & 40 & 57,6 & 6,4 & 38,2 \\
\hline
\end{tabular}

Parcelas com extraçāo seletiva de madeira

\begin{tabular}{lccccc}
\hline Bloco I & $0-5$ & 5 & 3,7 & 0,7 & 1,6 \\
& 10 & 5 & 6,7 & 1,3 & 3,2 \\
& $\mathbf{2 0}$ & $\mathbf{1 0}$ & 12,0 & 2,2 & $\mathbf{6 , 9}$ \\
& $\mathbf{3 0}$ & $\mathbf{1 0}$ & 18,3 & 3,2 & $\mathbf{1 0 , 8}$ \\
& $\mathbf{4 0}$ & 10 & 24,0 & 4,2 & $\mathbf{1 4 , 0}$ \\
Bloco II & 60 & 20 & 35,6 & 6,2 & $\mathbf{2 0 , 6}$ \\
& 100 & $\mathbf{4 0}$ & 58,1 & 10,6 & $\mathbf{3 5 , 0}$ \\
& 0.5 & $\mathbf{5}$ & 3,5 & 0,7 & $\mathbf{1 , 7}$ \\
& 10 & $\mathbf{5}$ & 6,3 & 1,1 & $\mathbf{3 , 7}$ \\
& $\mathbf{2 0}$ & 10 & 12,7 & 2,0 & $\mathbf{8 , 5}$ \\
& $\mathbf{3 0}$ & 10 & 18,3 & 2,9 & $\mathbf{1 2 , 1}$ \\
& $\mathbf{4 0}$ & $\mathbf{1 0}$ & 24,1 & 3,7 & $\mathbf{1 5 , 7}$ \\
& 100 & 60 & 57,8 & 9,7 & $\mathbf{3 7 , 3}$ \\
& $0-5$ & $\mathbf{5}$ & 3,4 & 0,6 & $\mathbf{1 , 7}$ \\
& $\mathbf{1 0}$ & $\mathbf{5}$ & 6,5 & $\mathbf{1 , 3}$ & $\mathbf{3 , 6}$ \\
& $\mathbf{2 0}$ & $\mathbf{1 0}$ & 12,4 & 2,3 & $\mathbf{7 , 2}$ \\
& $\mathbf{3 0}$ & 10 & 18,3 & 3,2 & 10,7 \\
& $\mathbf{4 0}$ & $\mathbf{1 0}$ & 23,9 & $\mathbf{4 , 3}$ & $\mathbf{1 4 , 2}$ \\
& $\mathbf{6 0}$ & $\mathbf{2 0}$ & $\mathbf{3 5 , 6}$ & $\mathbf{6 , 5}$ & $\mathbf{2 1 , 0}$ \\
& 100 & 40 & 58,5 & 10,5 & 35,8 \\
\hline
\end{tabular}


Tabela 3. Densidade do solo $\left(\mathrm{g} / \mathrm{cm}^{3}\right)$ nas parcelas-controle e nas extraidas seletivamente (manejadas) em 1993.

\begin{tabular}{lcccccc}
\hline & \multicolumn{3}{c}{ Parcelas controle } & \multicolumn{3}{c}{ Parcelas manejadas } \\
\hline Prof. $(\mathrm{cm})$ & Bloco I & Bloco II & Bloco IV & Bloco I & Bloco II & Bloco IV \\
\hline $0-5$ & 0,74 & 0,84 & nd & 0,67 & 0,87 & 0,91 \\
10 & 1,01 & 1,06 & 0,93 & 0,98 & 1,23 & 1,17 \\
20 & 1,10 & 1,02 & 0,99 & 1,15 & 1,13 & 1,08 \\
30 & 1,10 & 1,07 & nd & 1,19 & 1,11 & 1,05 \\
40 & 1,07 & 1,05 & 1,06 & 1,04 & 1,07 & 1,12 \\
60 & 1,09 & 1,05 & 1,16 & 1,04 & nd & 1,08 \\
100 & 1,16 & 1,23 & 1,21 & 1,12 & 1,11 & 1,13 \\
\hline nd-năo determinado & & & & & &
\end{tabular}

Corrêa (1984) para solos próximos da área estudada, onde os dados obtidos em pastagem e floresta sobre Latossolo Amarelo, textura argilosa, variaram na faixa de 0,9 a $1,5 \mathrm{~g} / \mathrm{cm}^{3}$, sendo para a floresta primária: no horizonte $A_{1}(0-8 \mathrm{~cm}), 0,9 \mathrm{~g} / \mathrm{cm}^{3} ; A_{3}$ $(8-33 \mathrm{~cm}), 1,1 \mathrm{~g} / \mathrm{cm}^{3}$ e $B_{2}(33-70 \mathrm{~cm})$, $1,2 \mathrm{~g} / \mathrm{cm}^{3}$. Medina \& Leite (1985) registraram valores bem mais elevados, de $1,26 \mathrm{~g} / \mathrm{cm}^{3}$ e $1,29 \mathrm{~g} / \mathrm{cm}^{3}$, respectivamente nas camadas $0-15 \mathrm{~cm}$ e $15-30 \mathrm{~cm}$ de um Latossolo Amarelo sob floresta primária.

O Latossolo Amarelo de textura muito argilosa, muito frequente na Amazônia Central, com ou sem saturação elevada de alumínio, é um solo profundo, bastante envelhecido, que apresenta baixo conteúdo de carbono orgânico. A capacidade de troca iônica nos horizontes superficiais é significantemente dominada pela matéria orgânica, que chega alcançar $82 \%$ da capacidade de troca catiônica total do solo (Vieira \& Santos, 1987).

No presente estudo, com a retirada das árvores, era de se esperar um aumento da densidade nas parcelas manejadas devido à ação do trator, e também à exposição do solo, mas as diferenças não foram significativas.

Condutividade hidráulica de solo saturado (Ko). A condutividade hidráulica do solo é uma propriedade essencial na análise da dinâmica da água no solo, pois representa a facilidade com que o solo é capaz de conduzir a água. Esta determinação também é importante para o entendimento do transporte de nutrientes no solo, pois este é estritamente dependente do fluxo de água. No presente estudo, o valor médio mais elevado de infiltração básica ou condutividade hidráulica saturada foi registrado no controle do bloco II $(42,7 \mathrm{~mm} / \mathrm{h})$ e o mais baixo na parcela manejada do bloco I $(11,3$ $\mathrm{mm} / \mathrm{h}$ ) (Tab. 4). Os altos desvios padrão indicam uma grande variabilidade espacial do solo, tornando difícil a constatação de eventuais diferenças, exceto na condutividade minima, claramente mais baixa nas parcelas com extração 
Tabela 4. Condutividade hidráulica de solo saturado $(\mathrm{Ko}, \mathrm{mm} / \mathrm{h})$ nas parcelas-controle e submetidas à extração seletiva de madeira, nos blocos I, II e IV da exploração de 1993 e do bloco IV da exploração de 1987.

\begin{tabular}{|c|c|c|c|c|c|}
\hline Locais & $\mathrm{N}$ & $\begin{array}{l}\text { Média } \\
\mathrm{mm} / \mathrm{h}\end{array}$ & s & $\begin{array}{c}\text { Máximo } \\
\mathrm{mm} / \mathrm{h}\end{array}$ & $\begin{array}{c}\text { Mínimo } \\
\mathrm{mm} / \mathrm{h}\end{array}$ \\
\hline \multicolumn{6}{|c|}{ Parcelas-controle } \\
\hline Bloco 1 & 5 & 40,0 & 36,0 & 106 & 7,1 \\
\hline Bloco II & 6 & 42,7 & 39,1 & 109 & 6,5 \\
\hline Bloco IV 93 & 4 & 25,3 & 13,3 & 47,5 & 12,2 \\
\hline Bloco IV 87 & 5 & 35,0 & 41,8 & 118 & 6,2 \\
\hline \multicolumn{6}{|c|}{ Parcelas com extraçăo seletiva } \\
\hline Bloco I & 8 & 11,3 & 12,6 & 31,9 & 2,3 \\
\hline Bloco II & 12 & 39,8 & 42,8 & 130 & 0,5 \\
\hline Bloco IV 93 & 10 & 32,1 & 26,5 & 79,1 & 0,4 \\
\hline Bloco IV 87 & 4 & 31,0 & 25,3 & 73,1 & 8,3 \\
\hline
\end{tabular}

seletiva de madeira de 1993 do que no controle.

Apesar da alta proporção de argila, muitas vezes acima de $80 \%$, o Latossolo Amarelo sob floresta apresenta elevada condutividade hidráulica saturada. Uma das características dos Latossolos é a microagregação entre as partículas do solo, que faz com que eles se comportem como alguns solos arenosos, em termos de apresentarem elevada condutividade. Além da distribuição de tamanho de agregados destes solos, mais de $50 \%$ dos agregados encontram-se em classes maiores que $2 \mathrm{~mm}$ (Mello, 1994). De acordo com Reichardt (1990), a infiltração básica acima de $30 \mathrm{~mm} / \mathrm{h}$ é considerada muito alta. Com exceção da parcela manejada em 1993 do Bloco I e o controle de 1993 do Bloco IV, os demais resultados estão acima desse valor, e a infiltração básica desses solos pode ser classificada de alta a muito alta. Medina \& Leite (1985) registraram valores bem mais elevados em Latossolo Amarelo próximo à Manaus. Eles avaliaram a influência de três sistemas de manejo do solo e de duas coberturas vegetais na infiltração de água. O valor mais elevado que registraram foi $285 \mathrm{~mm} / \mathrm{h}$ para o sistema seringueira com cobertura de leguminosa e em floresta primária $(223 \mathrm{~mm} / \mathrm{h})$. Atribuiram a facilidade do solo em transmitir a água à ação granulante, estabilizadora e protetora da vegetação de cobertura, que induz o desenvolvimento de propriedades estruturais do solo que afetam a sua habilidade para transmitir fluidos. Por outro lado, o excessivo tráfego de máquinas agrícolas, que compactou o solo, e a ação dispersante e 
impermeabilizante da chuva, proporcionaram uma penetração da água muito lenta no solo sob outros tratamentos avaliados.

Tomasella \& Hodnett (1996) determinaram a infiltração básica em Latossolo Amarelo em três profundidades (superfície, 30 e 105 $\mathrm{cm}$ ) em uma pastagem formada em 1980 a $100 \mathrm{~km}$ de Manaus (Fazenda Dimona). Observaram que a infiltração na superfície $(66 \mathrm{~mm} / \mathrm{h})$ foi mais baixa do que a $30 \mathrm{~cm}(97 \mathrm{~mm} /$ h). Na profundidade $0-5 \mathrm{~cm}$, o valor obtido foi $17 \mathrm{~mm} / \mathrm{h}$. Afirmam que os altos valores de Ko nas camadas superiores são devidos à elevada macroporosidade. Reichardt et al. (1980) determinaram, em laboratório, os valores de Ko em Latossolo Amarelo da Estação Experimental de Silvicultura Tropical do INPA, a cerca de $60 \mathrm{~km}$ de Manaus, obtendo um valor médio de $35 \mathrm{~mm} / \mathrm{h}$.

Portanto, os resultados aqui apresentados confirmaram os encontrados por outros pesquisadores, de que o Latossolo Amarelo de textura muito argilosa tem elevada condutividade hidráulica saturada, o que, aliada à sua boa porosidade, acima de $50 \%$, proporciona uma boa infiltração e drenagem neste tipo de solo. Durante as coletas, algumas vezes sob intensa chuva, nunca foi observado escoamento superficial nos blocos investigados, ou seja, toda a água que chegou ao solo infiltrou.

Temperatura do solo. As temperaturas do solo, a $5 \mathrm{~cm}$ de profundidade, foram medidas considerando-se os cinco níveis de distúrbios da extração seletiva de madeira. O valor mais baixo da temperatura do solo a $5 \mathrm{~cm}$ de profundidade $\left(22,6{ }^{\circ} \mathrm{C}\right)$ foi registrado no tratamento trilha do trator do bloco II em 08/09/1994, e o mais elevado $\left(35^{\circ} \mathrm{C}\right)$ registrado na borda da clareira do bloco II em 06/12/94. No mesmo dia em que foi registrado este valor mais elevado de temperatura do solo, observou-se, uma diferença de $9,4{ }^{\circ} \mathrm{C}$ em relação à medida feita na parcela controle. Na profundidade de $10 \mathrm{~cm}$ (dados não-mostrados), registrou-se o valor mais baixo $\left(22,2^{\circ} \mathrm{C}\right)$ no controle do bloco I, e o mais elevado $\left(30,4^{\circ} \mathrm{C}\right)$ no centro da clareira do bloco I

Quando se emprega o gradiente, algumas vezes o entendimento do sentido do fluxo de calor é confuso; no caso da temperatura ser mais elevada na superfície em relação à temperatura em profundidade abaixo, o gradiente é negativo e há um fluxo de calor de cima para baixo. Nota-se que os gradientes mais negativos ocorreram nas parcelas com extração seletiva (Tab. 5) e o valor mais expressivo foi $-1,6{ }^{\circ} \mathrm{C} / \mathrm{cm}$ (bloco $\mathrm{II}$, centro da clareira). Também foram encontrados valores mais elevados de temperatura na profundidade de $10 \mathrm{~cm}$, indicando arrefecimento da camada mais superficial, e, nesse caso, o gradiente de temperatura foi positivo. Nas parcelascontrole, o solo até a profundidade de $10 \mathrm{~cm}$ está sujeito a um baixo fluxo de calor, enquanto que nas clareiras, bordas de clareira e bordas da floresta remanescente, o gradiente de temperatura e o fluxo são mais intensos. 
Tabela 5. Gradientes de temperatura no perfil do solo $\left({ }^{\circ} \mathrm{C} / \mathrm{cm}\right)$ nas parcelas-controle e nos centros de clareira (CC) dos Bloco I, II e IV e na borda de clareira (BC) do Bloco II.

\begin{tabular}{|c|c|c|c|c|c|c|c|}
\hline \multirow[b]{2}{*}{ Data } & \multicolumn{2}{|c|}{ Bloco I } & \multicolumn{2}{|c|}{ Bloco II } & \multicolumn{3}{|c|}{ Bloco IV } \\
\hline & Controle & $\mathrm{CC}$ & Controle & $\mathrm{CC}$ & $\mathrm{BC}$ & Controle & $\mathrm{CC}$ \\
\hline $10 / 02 / 94$ & $-0,1$ & $-0,2$ & 0.1 & $-0,4$ & $.0,6$ & $-0,1$ & $-0,1$ \\
\hline $23 / 02 / 94$ & $-0,1$ & $\cdot 0.2$ & 0,0 & $-0,4$ & $-0,2$ & 0.0 & 0.0 \\
\hline $01 / 03 / 94$ & $-0,1$ & $-0,3$ & $-0,1$ & $-0,2$ & $\mathrm{~nm}$ & 0,0 & $-0,1$ \\
\hline $08 / 03 / 94$ & $-0,1$ & $-0,6$ & 0,0 & $-0,8$ & $-0,2$ & $-0,1$ & $-0,1$ \\
\hline $22 / 03 / 94$ & $-0,2$ & $-1,0$ & 0,0 & $-1,0$ & $-0,4$ & 0,0 & $-0,1$ \\
\hline $06 / 04 / 94$ & $-0,2$ & $-1,2$ & $-0,1$ & $-0,9$ & -0.3 & -0.1 & $-0,5$ \\
\hline $12 / 04 / 94$ & $-0,1$ & $.0,4$ & 0,0 & $-1,0$ & -0.3 & $-0,1$ & $-0,2$ \\
\hline $19 / 04 / 94$ & $\mathrm{~nm}$ & $-0,8$ & 0,1 & $-0,6$ & $-0,1$ & $-0,1$ & 0,0 \\
\hline $03 / 05 / 94$ & $-0,1$ & $-0,2$ & $-0,1$ & $-0,6$ & $-0,2$ & 0,0 & $-0,1$ \\
\hline $18 / 05 / 94$ & $\mathrm{~nm}$ & $-0,2$ & $-0,1$ & $-0,4$ & $-0,1$ & 0,0 & 0,0 \\
\hline $25 / 05 / 94$ & $-0,1$ & $-0,2$ & 0,0 & $-0,6$ & 0.0 & $-0,1$ & 0,0 \\
\hline $09 / 06 / 94$ & $-0,2$ & $-0,2$ & 0,0 & -0.9 & 0.0 & $\mathrm{Nm}$ & $\mathrm{nm}$ \\
\hline $15 / 06 / 94$ & $-0,2$ & $-0,6$ & $-0,1$ & $-1,6$ & 0,0 & $\mathrm{Nm}$ & 0,1 \\
\hline $29 / 06 / 94$ & $-0,2$ & -0.1 & 0,0 & $-0,4$ & $-0,2$ & 0,0 & 0,0 \\
\hline $06 / 07 / 94$ & $-0,1$ & $\mathrm{~nm}$ & $\mathrm{~nm}$ & $\mathrm{~nm}$ & $\mathrm{~nm}$ & 0.0 & -0.3 \\
\hline $20 / 07 / 94$ & 0,4 & -0.5 & 0.0 & $-1,0$ & 0,0 & 0.0 & 0,0 \\
\hline $03 / 08 / 94$ & $-0,2$ & $\cdot 0,4$ & $-0,1$ & $-1,1$ & $\mathrm{~nm}$ & $-0,1$ & $-0,3$ \\
\hline $17 / 08 / 94$ & $-0,2$ & $-0,3$ & 0,0 & $-0,8$ & 0,0 & $-0,1$ & $-0,2$ \\
\hline $26 / 08 / 94$ & $-0,3$ & $\mathrm{~nm}$ & 0,0 & $-0,7$ & $\mathrm{~nm}$ & 0,0 & $\mathrm{~nm}$ \\
\hline $31 / 08 / 94$ & $\mathrm{~nm}$ & $-0,3$ & 0,0 & $-0,5$ & $\mathrm{~nm}$ & $-0,1$ & $-0,1$ \\
\hline $08 / 09 / 94$ & 0,0 & $-0,1$ & 0,0 & 0,0 & $-0,2$ & 0,1 & $-0,1$ \\
\hline $14 / 09 / 94$ & $-0,3$ & $\mathrm{~nm}$ & $\mathrm{~nm}$ & $-0,7$ & $-0,1$ & $-0,1$ & $-0,2$ \\
\hline $22 / 09 / 94$ & $-0,2$ & 0,2 & 0,0 & $-1,2$ & 0,0 & $-0,1$ & $-0,2$ \\
\hline $30 / 09 / 94$ & $-0,2$ & $\cdot 0,3$ & 0,0 & $-0,4$ & $-0,1$ & 0,0 & $-0,3$ \\
\hline $06 / 10 / 94$ & $-0,2$ & 0,0 & 0,1 & 0,0 & $\mathrm{~nm}$ & 0,0 & $-0,2$ \\
\hline $26 / 10 / 94$ & $-0,1$ & 0,0 & $-0,1$ & $-0,2$ & 0,0 & $-0,1$ & $-0,2$ \\
\hline $03 / 11 / 94$ & $-0,2$ & -0.1 & 0,0 & $-0,8$ & $\mathrm{~nm}$ & -0.1 & 0,0 \\
\hline $09 / 11 / 94$ & $-0,2$ & 0,0 & 0.1 & 0,0 & $-0,1$ & 0,0 & $-0,2$ \\
\hline $16 / 11 / 94$ & $-0,1$ & $-1,0$ & 0,0 & $-0,4$ & $\mathrm{~nm}$ & $-0,1$ & $-0,1$ \\
\hline $22 / 11 / 94$ & $-0,1$ & $\cdot 0,2$ & 0,0 & $-0,1$ & $-0,4$ & $-0,1$ & $-0,2$ \\
\hline $29 / 11 / 94$ & $-0,2$ & $-0,1$ & 0,0 & $-0,4$ & $\mathrm{~nm}$ & $-0,1$ & $-0,1$ \\
\hline $06 / 12 / 94$ & $-0,3$ & 0.1 & 0,0 & $-0,4$ & $-1,5$ & 0,0 & $-0,2$ \\
\hline $12 / 12 / 94$ & $-0,2$ & 0,0 & 0,0 & $-0,6$ & $-0,7$ & $-0,1$ & $-0,1$ \\
\hline $19 / 12 / 94$ & $-0,4$ & 0,0 & 0,0 & $-0,4$ & -0.2 & 0,0 & $-0,1$ \\
\hline $28 / 12 / 94$ & $-0,2$ & $\mathrm{~nm}$ & 0,0 & $-0,4$ & $-0,7$ & $-0,1$ & 0,0 \\
\hline $10 / 01 / 95$ & $-0,1$ & $-0,1$ & 0,0 & 0,0 & 0,0 & 0,1 & $-0,2$ \\
\hline $18 / 01 / 95$ & $-0,2$ & $-0,2$ & 0,0 & $-1,0$ & $-1,1$ & $-0,1$ & $-0,1$ \\
\hline $24 / 01 / 95$ & $-0,2$ & 0,0 & 0,0 & $-0,1$ & $\mathrm{~nm}$ & 0,0 & 0.0 \\
\hline $31 / 01 / 95$ & $-0,2$ & $-0,2$ & 0,0 & $-0,4$ & $\mathrm{~nm}$ & $-0,2$ & 0,0 \\
\hline $13 / 02 / 95$ & $-0,2$ & 0,0 & 0,0 & $-0,2$ & 0,0 & $-0,1$ & 0,0 \\
\hline $21 / 02 / 95$ & $-0,2$ & $-0,4$ & $\mathrm{~nm}$ & 0,0 & 0,0 & 0,0 & $-0,2$ \\
\hline
\end{tabular}

$\mathrm{nm}$ : Năo medido 
Souza et al. (1996) estudaram as variações de temperatura em perfís do solo até a profundidade de $3,8 \mathrm{~m}$ em dois sítios na região de Marabá, no Pará, sendo um sob floresta primária e o outro sob pastagem. Observaram que a temperatura sob pastagem foi $3,7{ }^{\circ} \mathrm{C}$ mais elevada do que sob floresta à profundidade de $0,20 \mathrm{~m}$. Durante o período chuvoso, considerando todas as profundidades que investigaram, as temperaturas sob pastagem foram em torno de $2{ }^{\circ} \mathrm{C}$ mais elevadas do que sob floresta.

No presente estudo, supostamente o centro das clareiras deveria sempre apresentar os valores mais elevados de temperatura, mas nos blocos I e IV, algumas vezes, isto não ocorreu, possivelmente devido à regeneração rápida da vegetação, que protegeu a superfície do solo e proporcionou valores mais baixos do que na borda de clareira. Com base nos resultados de temperatura do solo, é possível afirmar que a perda da cobertura florestal, que mantém o solo protegido de elevadas variações de temperaturas e de fluxos de calor, causou aumentos de temperaturas, de seus gradientes e de fluxo de calor nas clareiras formadas pela extração seletiva de madeira, especialmente nos centro de clareiras com menor proteção da vegetação.

Os dados de temperatura do solo para a profundidade $5 \mathrm{~cm}$ foram separados em três períodos (estações) para análise estatística, a saber: 1 . Chuvoso, de janeiro a junho/ $94 ; 2$. Seco, de julho a novembro/94 e 3. Chuvoso, de dezembro/94 a fevereiro/95. A Análise estatística foi feita através de análise de Variância. As diferentes classes foram comparadas pelo teste de Tukey, para a temperatura registrada na profundidade do solo $5 \mathrm{~cm}$, mostrou diferença significativa ao nível de $5 \%$ entre os tratamentos, nos três períodos; em relação aos blocos, apenas no primeiro período (chuvoso, de janeiro a junho/94) houve diferença significativa. Com exceção da floresta remanescente, que mostrou valores próximos aos do controle, as demais classes de dano mostraram temperaturas mais altas, com as mais elevadas registradas nas classes $\mathrm{CC}$ e $\mathrm{BC}$, sempre significativamente maiores do que nas classes BFR, FR e controle (Tab. 6).

Os dados de temperatura na profundidade $10 \mathrm{~cm}$ foram comparados apenas entre os tratamentos controle e centro da clareira. A Análise de Variância seguida do teste de Tukey indicaram diferenças significativas entre as médias do controle e do centro da clareira, para os três periodos climáticos estudados (Tab. 7). Portanto, o corte seletivo de árvores, com a formação de clareiras e de suas bordas, causou aumentos de temperatura na camada superior do solo, até $10 \mathrm{~cm}$, o que pode influenciar diversos processos no solo, incluindo a ciclagem de nutrientes.

\section{Conclusões}

As curvas de retenção da água do solo mostraram que a camada mais superficial $(0-10 \mathrm{~cm})$ tem uma porosidade mais elevada e tende a dispor de mais água às plantas. Num perfil de $1 \mathrm{~m}$ de profundidade, somente cerca de 11 a $18 \%$ de água desses solos podem estar disponiveis 
Tabela 6. Teste de Tukey entre os diferentes niveis de dano para a temperatura do solo na profundidade $5 \mathrm{~cm}$, considerando três períodos climáticos. $\mathrm{CC}=$ centro de clareira, $\mathrm{BC}=$ borda de clareira, $\mathrm{BFR}=$ borda da floresta remanescente, $\mathrm{FR}=$ floresta remanescente e $\mathrm{TT}=$ trilha do trator,

\begin{tabular}{lccc}
\hline & Chuvoso/94 & seco/94 & chuvoso/95 \\
\hline Controle & $24,6 \mathrm{e}$ & $25,0 \mathrm{e}$ & $25,2 \mathrm{e}$ \\
CC & $29,0 \mathrm{a}$ & $28,4 \mathrm{~b}$ & $28,1 \mathrm{c}$ \\
BC & $27,7 \mathrm{~b}$ & $28,2 \mathrm{bc}$ & $29,4 \mathrm{~b}$ \\
BFR & $26,4 \mathrm{~cd}$ & $27,1 \mathrm{~d}$ & $27,0 \mathrm{~d}$ \\
FR & $25,1 \mathrm{de}$ & $25,4 \mathrm{e}$ & $25,8 \mathrm{e}$ \\
TT & $26,9 \mathrm{c}$ & $27,2 \mathrm{~cd}$ & $27,1 \mathrm{~cd}$ \\
\hline
\end{tabular}

Médias com mesma letra nas colunas não diferem entre si a $5 \%$ de probabilidade.

Tabela 7. Teste de Tukey, entre os tratamentos, para a temperatura do solo na profundidade 10 $\mathrm{cm}$, considerando três periodos climáticos para o controle e CC (centro de clareira).

\begin{tabular}{lccc}
\hline & janeiro - junho/94 & julho - novembro/94 & dezembro/94- fevereiro/95 \\
\hline Controle & $24,2 \mathrm{~b}$ & $24,6 \mathrm{~b}$ & $24,8 \mathrm{~b}$ \\
$\mathrm{CC}$ & $26,9 \mathrm{a}$ & $27,1 \mathrm{a}$ & $27,1 \mathrm{a}$ \\
\hline
\end{tabular}

Médias com letras diferentes nas colunas diferem entre si a $5 \%$ de probabilidade.

às plantas. A infiltração básica ou condutividade hidráulica saturada é elevada e os altos desvios-padrão indicam grande variabilidade espacial do solo. A extração seletiva de madeira não causou alterações acentuadas nas propriedades fisicas do solo, tais como a densidade do solo e a água disponivel no solo. No entanto, a retirada de árvores e a conseqüente formação de clareiras provocou um maior aquecimento das camadas superiores do solo, além de um maior fluxo de calor nas camadas superficiais do solo, o que pode afetar vários processos funcionais no solo.

\section{Agradecimentos}

Agradecemos a valiosa colaboração dos colegas José Geraldo
Martins dos Santos, Antônia Gomes Neta Pinto, João Augusto Dantas de Oliveira, Jonas de Oliveira Moraes e Sandoval do Nascimento Morais (INPA). Também ao Engo. Max Sarrazin (IRD da Guiana Francesa) e ao Folco H. H. H. van Rij.

\section{Bibliografia citada}

Camargo, M.N.; Rodrigues, P.E. 1979. Guia de excursão. XVII Congresso Brasileiro de Ciência do solo, Manaus. EMBRAPA, 72p.

Chauvel, A. 1982. Os latossolos amarelos, álicos, argilosos dentro dos ecossistemas das bacias experimentais do INPA e da região vizinha. Acta Amazonica, 12(3): 47-60.

Chauvel, A.; Vital, A.R.T.; Lucas, Y.; Desjardins, T.; Franken, W.; Luizào, F.J.; A-Araguás, L.; Rozanski, K. e Bedmar, A.P. 1992. O papel das raizes no ciclo hidrológico da floresta Amazônica. In: 
Anais do VII Congresso Brasileiro de Meteorologia. São Paulo. p. 29-37.

Corrêa, J.C. 1984. Características físico-hidricas dos solos latossolo amarelo, podzólico vermelho-amarelo e podzol hidromórfico do Estado do Amazonas. Pesq. agropec. Bras., Brasilia, 19(3): 347-360.

Dourado-Neto, D.; Nielsen, D.; Hopmans, J.W.; Parlange, M.B. Programa SWRC (versấo $2.00 p$ ): curva de retençào. Piracicaba, 1996 /Disquete.

Ferreira, S.J.F. 1993. Caracterização de sedimentos e solos de dois lagos de várzea na Amazonia Central. Piracicaba. Dissertação (Mestrado). Centro de Energia Nuclear na Agricultura, Universidade de Sào Paulo. $111 \mathrm{p}$.

Grimaldi, M.; Sarrazin, M; Chauvel, A.; Luizào, F.; Nunes, N.; Rodrigues, M.R.L.; Amblard, P; Tessier, D. 1993. Effets de la déforestation et des cultures sur la struture des sols argileux d'Amazonie brésilienne. Cahiers Agricultures, 2: 36-47.

Higuchi, N; Vieira, G.; Minete, L.J.; Freitas, J.V.; Jardim, F.C.S. 1991. Sistema S.E.L. (Seleção de Espécies Listadas) para manejar a floresta tropical úmida de terra firme da Amazônia. In: Val, A.L.; Figlioulo, R.; Feldberg, E.(Eds). Bases cientificas para estratégias de preservação e desenvolvimento $d a$ Amazônia. Vol 1. Instituto Nacional de Pesquisas da Amazônia (INPA). Manaus, Amazonas. p. 197-206.

Medina, B.F,; Leite, J.A. 1985. Influência de três sistemas de manejo e duas coberturas vegetais na infiltração de água em um latossolo amarelo em Manaus-Am. . Pesq. agropec, Bras., 20(11): 1323-1331.

Mello, W. 1994. Efeito da derrubada seletiva de madeira na estrutura de um latossolo sob floresta de terra firme na amazônia central. In: Resumos da X Reuniào Brasileira de Manejo e Conservação do Solo e da Água, Forianópolis. p. 380.

Nortcliff, S; Dias, A.C.D.C. 1988. The change in soil physical conditions resulting from forest clearance in the humic tropics. Journal of Biogeography, 15: 61-66.

Reichardt, K.; Razani, G.; Freitas J.R., E.; Libardi,
P.L. 1980. Aspectos hídricos de alguns solos da Amazônia - Região do baixo rio Negro. Acta Amazonica, 10(1): 43-46.

Reichardt, K. 1988. Capacidade de Campo. Revista Brasileira de Ciência do Solo, 12: 211-216.

Reichardt, K. 1990. A água em sistemas agricolas, São Paulo, Editora Manole. 188p.

Reynolds, W.D.; Elrick, D.E. 1985. In situ measurement of field-saturated hydraulic conductivity, sorptivity, and the a-parameter using the Guelph permeameter. Soil Science, 140(4): 292-302.

Reynolds, W.D. 1993. Satured hydraulic conductivity: field measurement. In: Carter, M.R. (ed), Soil sampling and methods of analysis. Canada, Lewis, p. 599-613.

Souza, J.R.S.; Pinheiro, F.M.A.; Araújo, R.L.C.; Pinheiro Jr., H,S.; Hodnett, M.G. 1996. Temperature and moisture profiles in soil beneath forest and pasture areas in eastern Amazonia. In: Gash, J.H.C.; Nobre, C.A.; Roberts, J.M.; Victoria, R.L. (Eds). Amazonian deforestation and climate. Chichester, John Wiley. p. 125-137.

Tomasella, J.; Hodnett, M.G. 1996. Soil hydraulic properties and Van Genuchten parameters for an oxisol under pasture in Central Amazonia. In: Gash, J.H.C.; Nobre, C.A.; Roberts, J.M.; Victoria, R.L. (Eds). Amazonian deforestation and climate. Chichester, John Wiley. p. 101-124.

Uhl, C; Almeida, O. 1996. O desafio da exploração sustentada na Amazônia. In: Almeida, O.T. (Ed). A evolução da fronteira Amazônica: oportunidade para um desenvolvimento sustentável. Belém, IMAZON. p. 1-6.

Vieira, L.S.; Santos, P.C.T.C. 1987. Amazônia: seus solos e outros recursos naturais. São Paulo, Ed. Agronômica Ceres. 416p.

Van Genuchten, M. 1980. A closed-form equation for predicting the hidraulic conductivity of unsaturated soils. Soil Science Society of American Journal, Madison, 44: 892-898.

Aceito para publicação em 19/06/2002 